\title{
ON DEGENERACY SCHEMES OF MAPS OF VECTOR BUNDLES AND APPLICATIONS TO HOLOMORPHIC FOLIATIONS
}

\author{
CAROLINA ARAUJO AND MAURÍCIO CORRÊA JR.
}

\begin{abstract}
In this paper we provide sufficient conditions for maps of vector bundles on smooth projective varieties to be uniquely determined by their degeneracy schemes. We then specialize to holomorphic distributions and foliations. In particular, we provide sufficient conditions for foliations of arbitrary rank on $\mathbb{P}^{n}$ to be uniquely determined by their singular schemes.
\end{abstract}

\section{INTRODUCTION}

This work was motivated by the following problem:

When is a holomorphic foliation uniquely determined by its singular scheme?

This problem has its origins in the study of spaces of foliations. When the general member of a fixed irreducible component of a space of foliations is determined by its singular scheme, the Hilbert scheme becomes a useful tool for describing such component. In this case, one hopes to read off properties of foliations from geometric aspects of their singular schemes.

A rank $k$ holomorphic foliation $\mathscr{F} \subset T_{X}$ on a complex projective manifold $X$ gives rise to a global section $\omega \in H^{0}\left(X, \bigwedge^{k} T_{X} \otimes\left(\bigwedge^{k} \mathscr{F}\right)^{*}\right)$. The singular scheme of $\mathscr{F}$ is precisely the zero scheme of $\omega$. The first observation is that $\mathscr{F}$ can be recovered from the global section $\omega$ (see Remark 3.3). So we are reduced to the classical problem of reconstructing sections of locally free sheaves from their zero schemes. We refer to Har78 for a general discussion on this topic. Often one cannot determine a section of $\bigwedge^{k} T_{X} \otimes\left(\bigwedge^{k} \mathscr{F}\right)^{*}$ by its zero scheme. However, we show that, under suitable conditions, this can be done for sections arriving from foliations.

The same approach can be used to tackle the following more general problem:

When is a map of vector bundles on a smooth projective variety determined by its degeneracy scheme?

Degeneracy schemes of maps of vector bundles show up in a number of geometric constructions. Example 2.4 discusses an instance of this, which has been classically studied by Castelnuovo, Palatine and Fano, among others.

1991 Mathematics Subject Classification. 14F05, 32S65, 37F75. 
We adopt the following notation. Given a locally free sheaf $\mathscr{E}$ of rank $e$ on a variety $X$, we denote by $\mathscr{E}^{*}$ its dual sheaf, by $S_{i} \mathscr{E}$ its $i$-th symmetric power, and by $\operatorname{det}(\mathscr{E})$ the invertible sheaf $\bigwedge^{e} \mathscr{E}$. In this paper we prove the following criterion, which holds for varieties defined over an arbitrary algebraically closed field.

Theorem 1.1. Let $X$ be a smooth projective variety, and $\mathscr{E}$ and $\mathcal{G}$ locally free sheaves on $X$ of ranke and $g$, respectively. Let $\varphi: \mathscr{E} \rightarrow \mathcal{G}$ be a generically surjective morphism, denote by $\omega_{\varphi} \in H^{0}\left(X, \bigwedge^{g}\left(\mathscr{E}^{*}\right) \otimes \operatorname{det}(\mathcal{G})\right)$ the associated global section, and by $Z$ its zero scheme. Suppose that the following conditions hold.

- $Z$ has pure codimension $e-g+1$, and

- for every $i \in\{1, \ldots, e-g\}$,

$$
H^{i}\left(X, \bigwedge^{g}\left(\mathscr{E}^{*}\right) \otimes \bigwedge^{g+i} \mathscr{E} \otimes S_{i}\left(\mathcal{G}^{*}\right)\right)=0 .
$$

If $\omega \in H^{0}\left(X, \bigwedge^{g}\left(\mathscr{E}^{*}\right) \otimes \operatorname{det}(\mathcal{G})\right)$ is such that $\left.\omega\right|_{Z}=0$, then there is an endomorphism $\alpha \in \operatorname{End}\left(\bigwedge^{g}\left(\mathscr{E}^{*}\right)\right)$ such that $\omega=\alpha \circ \omega_{\varphi}$.

When $\mathscr{E}$ is fixed, the vanishing assumption of Theorem 1.1 holds provided that $\mathcal{G}$ is sufficiently positive (see Corollary 2.3 for a precise statement). In particular, if $\mathscr{F} \subset T_{X}$ is a sufficiently negative locally free distribution of rank $k$ with singular scheme of pure dimension $k-1$, and if the vector bundle $\bigwedge^{k} T_{X}$ is simple, then the inclusion $\mathscr{F} \hookrightarrow T_{X}$ is uniquely determined by its singular scheme. We remark that a similar approach was used in $\mathrm{CO} 03$ to determine foliations by curves by their singular schemes.

For complex projective spaces, the problem of determining holomorphic foliations by their singular schemes has been addressed by several authors. In G-MK89, Gomez-Mont and Kempf proved that a foliation by curves $\mathscr{F}$ on $\mathbb{P}^{n}$ corresponding to a global section of $T_{\mathbb{P}^{n}} \otimes \mathcal{O}_{\mathbb{P}^{n}}(r)$ is uniquely determined by its singular scheme $\operatorname{Sing}(\mathscr{F})$, provided that $r>0$ and $\operatorname{Sing}(\mathscr{F})$ is reduced. In CO01, Campillo and Olivares showed that when $n=2$ the hypothesis that $\operatorname{Sing}(\mathscr{F})$ is reduced may be removed. For $n \geq 3$, they showed in $\mathrm{CO} 03$ that $\mathscr{F}$ is uniquely determined by its singular scheme provided that $r>0$ and $\operatorname{Sing}(\mathscr{F})$ is zero-dimensional.

For foliations of rank higher than 1, much less is known. Giraldo and PanCollantes showed in GP-C10 that if $\mathscr{F} \subset T_{\mathbb{P}^{3}}$ is a rank 2 holomorphic foliation on $\mathbb{P}^{3}$ of the form $\mathscr{F} \cong \mathcal{O}_{\mathbb{P} n}(a) \oplus \mathcal{O}_{\mathbb{P} n}(b)$, with $a, b \leq-1$, then $\mathscr{F}$ is uniquely determined by its singular scheme. Using Theorem 1.1 and vanishing results of Manivel, we provide sufficient conditions for distributions of arbitrary rank on $\mathbb{P}^{n}$ to be uniquely determined by their singular schemes, recovering and generalizing Giraldo and Pan-Collantes' criterion. (See also Theorems 4.2 and 4.3.)

Theorem 1.2. Let $\mathscr{F} \subset T_{\mathbb{P}^{n}}$ be a holomorphic distribution of rank $k$ on $\mathbb{P}^{n}$ with singular scheme of pure dimension $k-1$. Suppose that one of the following conditions hold: 
(1) $\mathscr{F}$ is locally free and $\mathscr{F}^{*} \otimes \mathcal{O}_{\mathbb{P}^{n}}(k-n)$ is ample, or

(2) $\mathscr{F}$ decomposes as a direct sum of line bundles and $\mathscr{F}^{*} \otimes \mathcal{O}_{\mathbb{P}^{n}}(k-n+1)$ is ample.

Then $\mathscr{F}$ is uniquely determined by its singular scheme.

The assumptions of Theorem 1.2 are verified in several important cases (see Remmark 3.6). The next example illustrates the necessity of our hypothesis when $k=n-1$.

Example 1.3. Fix homogeneous polynomials $F_{0}, \cdots, F_{m} \in \mathbb{C}\left[x_{0}, \cdots, x_{n}\right]$, with degrees $d_{i}=\operatorname{deg}\left(F_{i}\right)>0$. Suppose that the hypersurfaces $\left\{F_{i}=0\right\}$ are smooth and in general position. Set $\Lambda=\left\{\left(\lambda_{0}, \cdots, \lambda_{m}\right) \in\left(\mathbb{C}^{*}\right)^{m+1} \mid \sum_{i=0}^{m} \lambda_{i} d_{i}=0\right\}$. For each $\lambda \in \Lambda$, we get a foliation $\mathscr{F}_{\lambda}$ of codimension 1 on $\mathbb{P}^{n}$ defined by the 1 -form

$$
\omega_{\lambda}=\sum_{i=0}^{m} \lambda_{i} F_{0} \cdots \widehat{F}_{i} \cdots F_{m} d F_{i}
$$

One can check that $\mathscr{F}_{\lambda} \neq \mathscr{F}_{\nu}$ if $\lambda, \nu \in \Lambda$ are not proportional.

By [CSV06, Theorem 3], $\operatorname{Sing}\left(\mathscr{F}_{\lambda}\right)$ has pure codimension 2 if and only if the following condition holds:

$$
m \leq n \text { and } d_{i}=1 \text { for all } i .
$$

If (11) does not hold, then $\operatorname{Sing}\left(\mathscr{F}_{\lambda}\right)$ contains isolated points, and thus $\mathscr{F}_{\lambda}$ is not locally free. If (11) holds, then $\operatorname{Sing}\left(\mathscr{F}_{\lambda}\right)=\cup_{i \neq j}\left\{F_{i}=F_{j}=0\right\}$ for all $\lambda \in \Lambda$. In this case, $\mathscr{F}_{\lambda} \cong \mathcal{O}_{\mathbb{P}^{n}}^{\oplus m-1} \oplus \mathcal{O}_{\mathbb{P}^{n}}(1)^{\oplus n-m}$, and $\mathscr{F}_{\lambda}^{*}$ is not ample.

By Jou79, Theorem 2.3], a generic distribution $\mathscr{F}$ of codimension 1 on $\mathbb{P}^{n}$ with $\operatorname{deg}(\mathscr{F})<n-1$ has zero dimensional singular scheme. On the other hand, by Jou79, Theorem 2.6], if $\mathscr{F}$ is a foliation, then its singular scheme has an irreducible component of codimension 2. Thus, when $n>2$ and $k=n-1$, Theorem 1.2 does not apply to generic distributions, but rather to foliations. However, the same methods allow us to show that generic codimension 1 distributions on $\mathbb{P}^{n}$ with negative degree are uniquely determined by their singular schemes.

Theorem 1.4. Let $\mathscr{F} \subset T_{\mathbb{P}^{n}}$ be a holomorphic distribution of rank $n-1$ on $\mathbb{P}^{n}$ with zero dimensional singular scheme. If $\operatorname{deg}\left(\mathscr{F}^{*}\right)>0$, then $\mathscr{F}$ is uniquely determined by its singular scheme.

This paper is organized as follows. In Section 2 we address the problem of determining maps of vector bundles by their degeneracy schemes. In Section 3 we review the notions of distribution, foliation and Pfaff field. In Section 4 we apply the results of Section 2 to the problem of recovering holomorphic distributions from their singular schemes. 
Notation. We identify a vector bundle $\mathscr{E}$ on a variety $X$ with its locally free sheaf of sections. When $X=\mathbb{P}^{n}$ and $m$ is an integer, we denote by $\mathscr{E}(m)$ the twisted sheaf $\mathscr{E} \otimes \mathcal{O}_{\mathbb{P}^{n}}(m)$.

Acknowledgements. The first named author was partially supported by CNPq and Faperj Research Fellowships. We would like to thank Fernando Cukierman for useful comments on an earlier version of this paper.

\section{Degeneracy SCheme of MAPS OF Vector BUndLes}

In this section all varieties are defined over a fixed algebraically closed field $\mathbf{k}$ of arbitrary characteristic.

Let $X$ be a smooth projective variety, $\mathscr{E}$ and $\mathcal{G}$ locally free sheaves on $X$ of rank $e$ and $g$, respectively, and $\varphi: \mathscr{E} \rightarrow \mathcal{G}$ a generically surjective morphism. The induced map $\wedge^{g} \varphi: \wedge^{g} \mathscr{E} \rightarrow \operatorname{det}(\mathcal{G})$ corresponds to a global section $\omega_{\varphi} \in$ $H^{0}\left(X, \bigwedge^{g}\left(\mathscr{E}^{*}\right) \otimes \operatorname{det}(\mathcal{G})\right)$.

Definition 2.1. The degeneracy scheme $\operatorname{Sing}(\varphi)$ of the map $\varphi: \mathscr{E} \rightarrow \mathcal{G}$ is the zero scheme of the associated global section $\omega_{\varphi} \in H^{0}\left(X, \bigwedge^{g}\left(\mathscr{E}^{*}\right) \otimes \operatorname{det}(\mathcal{G})\right)$.

Next we introduce the key tool to prove Theorem 1.1

2.2 (The Eagon-Northcott resolution). Let the notation be as above, and suppose that $Z=\operatorname{Sing}(\varphi)$ has pure expected dimension, i.e., $Z$ has pure codimension equal to $e-g+1$. Then the structure sheaf of $Z$ admits a special resolution, called the Eagon-Northcott resolution (see for instance [Eis95, A2.6]):

$$
\begin{aligned}
0 \rightarrow & \bigwedge^{e} \mathscr{E} \otimes S_{e-g}\left(\mathcal{G}^{*}\right) \otimes \operatorname{det}\left(\mathcal{G}^{*}\right) \rightarrow \bigwedge^{e-1} \mathscr{E} \otimes S_{e-g-1}\left(\mathcal{G}^{*}\right) \otimes \operatorname{det}\left(\mathcal{G}^{*}\right) \rightarrow \ldots \\
& \rightarrow \bigwedge_{g+1}^{g} \mathscr{E} \otimes \mathcal{G}^{*} \otimes \operatorname{det}\left(\mathcal{G}^{*}\right) \rightarrow \bigwedge^{g} \mathscr{E} \otimes \operatorname{det}\left(\mathcal{G}^{*}\right) \rightarrow \mathcal{O}_{X} \rightarrow \mathcal{O}_{Z} \rightarrow 0 .
\end{aligned}
$$

Proof of Theorem 1.1. Let $\omega_{\varphi} \in H^{0}\left(X, \bigwedge^{g}\left(\mathscr{E}^{*}\right) \otimes \operatorname{det}(\mathcal{G})\right)$ be the global section associated to the generically surjective morphism $\varphi: \mathscr{E} \rightarrow \mathcal{G}$, and $Z$ its zero scheme. The assumption that $Z$ has pure codimension $e-g+1$ allows us to consider the Eagon-Northcott resolution of $\mathcal{O}_{Z}$ as described in 2.2. Tensorizing it by $\bigwedge^{g}\left(\mathscr{E}^{*}\right) \otimes$ $\operatorname{det}(\mathcal{G})$ we get

$$
\begin{gathered}
0 \rightarrow \bigwedge^{g}\left(\mathscr{E}^{*}\right) \otimes \bigwedge^{e} \mathscr{E} \otimes S_{e-g}\left(\mathcal{G}^{*}\right) \rightarrow \bigwedge^{g}\left(\mathscr{E}^{*}\right) \otimes \bigwedge^{e-1} \mathscr{E} \otimes S_{e-g-1}\left(\mathcal{G}^{*}\right) \rightarrow \ldots \\
\left.\rightarrow \bigwedge^{g}\left(\mathscr{E}^{*}\right) \otimes \bigwedge^{g+1} \mathscr{E} \otimes \mathcal{G}^{*} \rightarrow \bigwedge^{g}\left(\mathscr{E}^{*}\right) \otimes \bigwedge^{g} \mathscr{E} \rightarrow \bigwedge^{g}\left(\mathscr{E}^{*}\right) \otimes \operatorname{det}(\mathcal{G}) \rightarrow \bigwedge^{g}\left(\mathscr{E}^{*}\right) \otimes \operatorname{det}(\mathcal{G})\right|_{Z} \rightarrow 0 .
\end{gathered}
$$


Setting $\mathscr{M}_{i}:=\bigwedge^{g}\left(\mathscr{E}^{*}\right) \otimes \bigwedge^{i} \mathscr{E} \otimes S_{i-g}\left(\mathcal{G}^{*}\right)$ for $g \leq i \leq e$, the above sequence breaks into short exact sequences:

$$
\begin{gathered}
0 \rightarrow \mathscr{M}_{e} \rightarrow \mathscr{M}_{e-1} \rightarrow \mathscr{F}_{e-g} \rightarrow 0 \\
\vdots \\
0 \rightarrow \mathscr{F}_{i-g+2} \rightarrow \mathscr{M}_{i} \rightarrow \mathscr{F}_{i-g+1} \rightarrow 0 \\
\vdots \\
0 \rightarrow \mathscr{F}_{2} \rightarrow \mathscr{M}_{g} \rightarrow \mathscr{F}_{1} \rightarrow 0 \\
\left.0 \rightarrow \mathscr{F}_{1} \rightarrow \bigwedge^{g}\left(\mathscr{E}^{*}\right) \otimes \operatorname{det}(\mathcal{G}) \rightarrow\left(\bigwedge^{g}\left(\mathscr{E}^{*}\right) \otimes \operatorname{det}(\mathcal{G})\right)\right|_{Z} \rightarrow 0
\end{gathered}
$$

By assumption $\omega \in H^{0}\left(X, \bigwedge^{g}\left(\mathscr{E}^{*}\right) \otimes \operatorname{det}(\mathcal{G})\right)$ is such that $\left.\omega\right|_{Z}=0$. It follows from the sequence (5) that $\omega$ comes from an element in $H^{0}\left(X, \mathscr{F}_{1}\right)$. We will show that $H^{1}\left(X, \mathscr{F}_{2}\right)=0$. It will then follow from the sequence (4) that there is an element $\alpha \in \operatorname{End}\left(\bigwedge^{g}\left(\mathscr{E}^{*}\right)\right) \cong H^{0}\left(\bigwedge^{g}\left(\mathscr{E}^{*}\right) \otimes \bigwedge^{g} \mathscr{E}\right)$ such that $\omega=\alpha \circ \omega_{\varphi}$.

By assumption

$$
H^{i}\left(X, \mathscr{M}_{g+i}\right)=0
$$

for $1 \leq i \leq e-g$. Applied to the cohomology of the sequence (3), these vanishings yield inclusions

$$
H^{i}\left(X, \mathscr{F}_{i+1}\right) \subset H^{i+1}\left(X, \mathscr{F}_{i+2}\right)
$$

for $1 \leq i \leq e-g-2$. On the other hand, applied the cohomology of the sequence (21), they give

$$
H^{e-g-1}\left(X, \mathscr{F}_{e-g}\right)=0 .
$$

By descending induction we get $H^{1}\left(X, \mathscr{F}_{2}\right)=0$, concluding the proof.

Corollary 2.3. Let $X$ be a smooth projective variety, $\mathscr{E}$ and $\mathcal{G}$ locally free sheaves on $X$ of rank e and $g$, respectively, and $\mathcal{L}$ an ample line bundle on $X$. There exists an integer $r_{0}$ such that, for every integer $r \geq r_{0}$, the following condition holds.

Let $\varphi: \mathscr{E} \rightarrow \mathcal{G} \otimes \mathcal{L}^{\otimes r}$ be a generically surjective morphism whose degeneracy scheme $Z=\operatorname{Sing}(\varphi)$ has pure codimension $e-g+1$. If

$$
\omega \in H^{0}\left(X, \bigwedge^{g}\left(\mathscr{E}^{*}\right) \otimes \operatorname{det}(\mathcal{G}) \otimes \mathcal{L}^{\otimes r g}\right)
$$

is such that $\left.\omega\right|_{Z}=0$, then there is an endomorphism $\alpha \in \operatorname{End}\left(\bigwedge^{g}\left(\mathscr{E}^{*}\right)\right)$ such that $\omega=\alpha \circ \omega_{\varphi}$. 
Proof. By Serre duality, $H^{i}\left(X, \bigwedge^{g}\left(\mathscr{E}^{*}\right) \otimes \bigwedge^{g+i} \mathscr{E} \otimes S_{i}\left(\mathcal{G}^{*}\right) \otimes \mathcal{L}^{* \otimes r i}\right)$ is isomorphic to $H^{\operatorname{dim}(X)-i}\left(X, \bigwedge^{g} \mathscr{E} \otimes \bigwedge^{g+i}\left(\mathscr{E}^{*}\right) \otimes S_{i} \mathcal{G} \otimes \omega_{X} \otimes \mathcal{L}^{\otimes r i}\right)$. It follows from Serre's vanishing theorem that there exists an integer $r_{0}$ such that the latter vanishes for every integer $r \geq r_{0}$ and every $i \in\{1, \ldots, e-g\}$. So we can apply Theorem 1.1.

Example 2.4. Let $m<n$ be positive integers and consider a general injective morphism

$$
\varphi: \mathcal{O}_{\mathbb{P}^{n}}^{\oplus m} \rightarrow \Omega_{\mathbb{P}^{n}}^{1}(2) .
$$

Denote by $Z$ the $(m-1)$-dimensional degeneracy scheme of $\varphi$, i.e., the degeneracy scheme of the induced map $T_{\mathbb{P}^{n}} \rightarrow \mathcal{O}_{\mathbb{P}^{n}}(2)^{\oplus m}$. These schemes have been studied by several classical algebraic geometers. Castelnuovo considered the case $m=3$ and $n=4$ in Cas91. In this case $Z$ is the projection in $\mathbb{P}^{4}$ of the Veronese surface. The case $m=3$ and $n=5$ was studied by Palatini in Pal01, and then by Fano in Fan30. In this case $Z$ is a scroll over an elliptic curve. Palatini considered the case $m=4$ and $n=5$ in Pal03. In this case $Z \subset \mathbb{P}^{5}$ is a scroll over a cubic surface in $\mathbb{P}^{3}$, known as Palatini scroll. More recently, Hilbert schemes of these degeneracy schemes were studied by Faenzi and Fania in [FF10.

When $m=n-1$, Theorem 1.1 implies that two general injective morphisms $\phi, \varphi: \mathcal{O}_{\mathbb{P}^{n}}^{\oplus(n-1)} \rightarrow \Omega_{\mathbb{P}^{n}}^{1}(2)$ have the same degeneracy scheme if and only if $\phi=\lambda \cdot \varphi$ for some $\lambda \in \mathbf{k}^{*}$. Indeed, by Bott's formulae (see 4.5),

$$
H^{1}\left(X, \Omega_{\mathbb{P}^{n}}^{n-1} \otimes \bigwedge^{n} T_{\mathbb{P}^{n}} \otimes \mathcal{O}_{\mathbb{P}^{n}}(-2)^{\oplus(n-1)}\right)=0,
$$

So Theorem 1.1 applies. Moreover, $\operatorname{End}\left(\Omega_{\mathbb{P} n}^{n-1}\right) \cong \mathbf{k}($ see Lemma 4.8) .

\section{Pfaff Fields, Distributions and Foliations}

In this section $X$ denotes a smooth complex projective variety of dimension $n$.

Definition 3.1. A Pfaff field of rank $k$ on $X$ is a nonzero map $\eta: \Omega_{X}^{k} \rightarrow \mathscr{L}$, where $\mathscr{L}$ is an invertible sheaf on $X$. It corresponds to a global section $\omega_{\eta} \in$ $H^{0}\left(X, \wedge^{k} T_{X} \otimes \mathscr{L}\right)$. The singular scheme $\operatorname{Sing}(\eta)$ of $\eta$ is the zero scheme of $\omega_{\eta}$.

Definition 3.2. A (holomorphic) distribution of rank $k$ on $X$ is a nonzero coherent subsheaf $\mathscr{F} \subsetneq T_{X}$ of generic rank $k$ which is saturated, i.e., such that $T_{X} / \mathscr{F}$ is torsion free.

If $\mathscr{F} \subsetneq T_{X}$ is a distribution of rank $k$ on $X$, then $\mathscr{F}$ is reflexive (see AD11, Remark 2.3]), and thus $\bigwedge^{k} \mathscr{F}$ is an invertible sheaf on $X$. So $\mathscr{F}$ naturaly gives rise to a Pfaff field of rank $k$ on $X$ :

$$
\eta_{\mathscr{F}}: \Omega_{X}^{k}=\bigwedge^{k}\left(T_{X}^{*}\right) \rightarrow\left(\bigwedge^{k} \mathscr{F}\right)^{*} .
$$

The singular scheme $\operatorname{Sing}(\mathscr{F})$ of $\mathscr{F}$ is defined to be the singular scheme of the associated Pfaff field. 
Note that when the distribution $\mathscr{F}$ is locally free, $\operatorname{Sing}(\mathscr{F})$ is precisely the degeneracy scheme of the dual map $\Omega_{X}^{1} \rightarrow \mathscr{F}^{*}$, as introduced in Definition 2.1.

Remark 3.3. Let $\mathscr{F} \subsetneq T_{X}$ be a distribution of $\operatorname{rank} k$ on $X$, and $\eta_{\mathscr{F}}: \Omega_{X}^{k} \rightarrow$ $\left(\bigwedge^{k} \mathscr{F}\right)^{*}$ the associated Pfaff field. Then $\mathscr{F}$ may be recovered from $\eta_{\mathscr{F}}$ as follows. As noted above, $\eta_{\mathscr{F}}$ corresponds to a global section $\omega \in H^{0}\left(X, \bigwedge^{k} T_{X} \otimes\left(\bigwedge^{k} \mathscr{F}\right)^{*}\right)$. Contraction with $\omega$ yields a morphism

$$
\imath(\omega): T_{X} \rightarrow \bigwedge^{k+1} T_{X} \otimes\left(\bigwedge^{k} \mathscr{F}\right)^{*} .
$$

Since it comes from a distribution, $\omega$ is locally decomposable. Therefore, the kernel of $\imath(\omega)$ is a saturated coherent subsheaf of $T_{X}$ of generic rank $k$. It is precisely the distribution $\mathscr{F}$. Here we use the assumption that $\mathscr{F}$ is saturated in $T_{X}$, and the fact that two saturated subsheaves of $T_{X}$ that coincide in a dense open subset must coincide.

Definition 3.4. A distribution on $X$ that is invariant under the Lie Bracket is called an involutive distribution. By abuse of notation, we call such distribution a (holomorphic) foliation on $X$.

Remark 3.5. Often in the literature, the definition of distribution and foliation does not require $\mathscr{F}$ to be saturated in $T_{X}$. What we call a foliation is often called a reduced foliation.

3.6 (Locally free foliations). Let $\mathscr{F}$ be a foliation of rank $k$ on $X$. We discuss sufficient conditions for $\mathscr{F}$ to be locally free.

If $k=1$, then $\mathscr{F}$ is always locally free. For $k \geq 2$, we introduce the Kupka condition. From the isomorphism $\bigwedge^{k} T_{X} \cong \Omega_{X}^{n-k} \otimes \omega_{X}^{*}$, we see that $\mathscr{F}$ corresponds to a twisted $(n-k)$-form $\omega \in H^{0}\left(X, \Omega_{X}^{n-k} \otimes \omega_{X}^{*} \otimes\left(\bigwedge^{k} \mathscr{F}\right)^{*}\right)$. We say that a point $P \in \operatorname{Sing}(\mathscr{F})$ is a Kupka singular point if $d \omega(P) \neq 0$. Geometrically, this means that, in an analytic neighborhood of $P, \mathscr{F}$ is equivalent to the product of a regular (and hence locally free) foliation and a foliation by curves. It follows that $\mathscr{F}$ is locally free at Kupka singular points (see dMe00, Proposition 1.3.1]).

For codimension 1 foliations, there are other useful conditions. First suppose that $n=3$ and $k=2$. A point $P \in \operatorname{Sing}(\mathscr{F})$ is a generalized Kupka (GK) singular point if either $d \omega(P) \neq 0$, or $P$ is an isolated singular point of $d \omega$. By [C-ACGLN04, Corollary 2], $\mathscr{F}$ is locally free at GK singular points. Moreover, by [C-ACGLN04, Corollary 1], the condition of having only GK singularities is open in the space of codimension 1 foliations on a smooth projective threefold. On the other hand, the condition of being locally free is not always open. The notion of GK singularity for codimension 1 foliations can be generalized to $n \geq 4$ as follows. A point $P \in$ $\operatorname{Sing}(\mathscr{F})$ is a GK singular point if there exists a threefold $Y \subset X$ smooth at $P$ such that either $d\left(\left.\omega\right|_{Y}\right)(P) \neq 0$, or $P$ is an isolated singular point of $d\left(\left.\omega\right|_{Y}\right)$ in $Y$. In this case it is not difficult to see that $\mathscr{F}$ is locally free at $P$. 
We also note that the condition of being a direct sum of line bundles is open in the space of codimension 1 foliations on $\mathbb{P}^{n}$ by [CP08, Theorem 1].

\section{RECOVERING HOLOMORPHIC DISTRIBUTIONS FROM THEIR SINGULAR SCHEMES}

Let $X$ be a smooth complex projective variety, and $\mathscr{F} \subsetneq T_{X}$ a locally free distribution. In order to recover $\mathscr{F}$ from $\operatorname{Sing}(\mathscr{F})$, we want to apply Theorem 1.1 to the dual map $\Omega_{X}^{1} \rightarrow \mathscr{F}^{*}$, and then Remark 3.3. So we need to establish the vanishing required in Theorem 1.1 .

In Man96, Manivel proved a number of vanishing results for varieties whose tangent bundle is uniformly nef.

Definition 4.1 ([Man96, p. 405]). The category of uniformly nef vector bundles on smooth complex projective varieties is the smallest category $\mathscr{C}$ containing tensor products of nef line bundles and Hermitian flat bundles, closed under direct sums, extensions, quotients, and satisfying the following property. If $f: Y \rightarrow X$ is a finite surjective morphism, and $\mathscr{E}$ is a vector bundle on $X$, then $\mathscr{E} \in \mathscr{C}$ if and only if $f^{*} \mathscr{E} \in \mathscr{C}$.

The class of smooth complex projective varieties having uniformly nef tangent bundle includes projective spaces, abelian varieties, products and finite étale covers of those, among others. For those varieties, we have the following result.

Theorem 4.2. Let $X$ be a smooth complex projective variety with uniformly nef tangent bundle, and $\mathscr{F} \subset T_{X}$ a locally free distribution of rank $k$ on $X$. Suppose that the following conditions hold.

- The singular scheme $\operatorname{Sing}(\mathscr{F})$ is of pure dimension $k-1$.

- There exists an ample line bundle $\mathscr{A}$ on $X$ such that $\mathscr{F}^{*} \otimes \mathscr{A}^{-1}$ is nef, and $\omega_{X} \otimes \mathscr{A}$ is ample.

Let $\eta: \Omega_{X}^{k} \rightarrow\left(\bigwedge^{k} \mathscr{F}\right)^{*}$ be a Pfaff field of rank $k$ on $X$, and suppose that $\operatorname{Sing}(\mathscr{F}) \subset$ $\operatorname{Sing}(\eta)$. Then there is an endomorphism $\alpha \in \operatorname{End}\left(\Omega_{X}^{k}\right)$ such that $\eta=\eta_{\mathscr{F}} \circ \alpha$.

When $X=\mathbb{P}^{n}$, we have the following more refined result.

Theorem 4.3. Let $\mathscr{F} \subset T_{\mathbb{P}^{n}}$ be a holomorphic distribution of rank $k$ on $\mathbb{P}^{n}$ with singular scheme $\operatorname{Sing}(\mathscr{F})$ of pure dimension $k-1$. Suppose that one of the following conditions hold:

(1) $\mathscr{F}$ is locally free and $\mathscr{F}^{*} \otimes \mathcal{O}_{\mathbb{P}^{n}}(k-n)$ is ample, or

(2) $\mathscr{F}$ decomposes as a direct sum of line bundles and $\mathscr{F}^{*} \otimes \mathcal{O}_{\mathbb{P}^{n}}(k-n+1)$ is ample.

Let $\eta: \Omega_{\mathbb{P} n}^{k} \rightarrow\left(\bigwedge^{k} \mathscr{F}\right)^{*}$ be a Pfaff field of rank $k$ on $\mathbb{P}^{n}$, and suppose that $\operatorname{Sing}(\mathscr{F}) \subset$ $\operatorname{Sing}(\eta)$. Then $\eta=\lambda \cdot \eta_{\mathscr{F}}$ for some $\lambda \in \mathbb{C}^{*}$.

The following result of Manivel provides the vanishing required to apply Theorem 1.1 in the context of Theorems 4.2 and 4.3 . 
Proposition 4.4 (Man96 p. 409]). Let $X$ be a smooth complex projective variety with uniformly nef tangent bundle. Let $\mathscr{L}$ be an ample line bundle on $X$, and $\mathscr{E}_{1}$ and $\mathscr{E}_{2}$ be nef vector bundles on $X$ of rank $r_{1}$ and $r_{2}$, respectively. Let $i$ and $j$ be positive integers. Then

$$
H^{p}\left(X, \Omega_{X}^{k} \otimes \bigwedge^{i} \mathscr{E}_{1} \otimes S_{j}\left(\mathscr{E}_{2}\right) \otimes \mathscr{L}\right)=0
$$

for every non-negative integer $k$ and every $p>r_{1}+r_{2}-i-1$.

Proof of Theorem 4.2. Since $\mathscr{F}$ is locally free, the singular scheme $Z:=\operatorname{Sing}(\mathscr{F})$ is the degeneracy scheme of the dual map $\Omega_{X}^{1} \rightarrow \mathscr{F}^{*}$. Since $Z$ has pure dimension $k-1$, the result will follow from Proposition 1.1 once we prove that

$$
H^{i}\left(X, \bigwedge^{k} T_{X} \otimes \Omega_{X}^{k+i} \otimes S_{i}(\mathscr{F})\right)=0
$$

for every $i \in\{1, \ldots, \operatorname{dim}(X)-k\}$.

By Serre duality,

$$
H^{i}\left(X, \bigwedge^{k} T_{X} \otimes \Omega_{X}^{k+i} \otimes S_{i}(\mathscr{F})\right) \cong H^{n-i}\left(X, \Omega_{X}^{k} \otimes \bigwedge^{k+i} T_{X} \otimes S_{i}\left(\mathscr{F}^{*}\right) \otimes \omega_{X}\right)
$$

Notice that

$$
S_{i}\left(\mathscr{F}^{*}\right) \otimes \omega_{X} \cong S_{i}\left(\mathscr{F}^{*} \otimes \mathscr{A}^{-1}\right) \otimes\left(\mathscr{A}^{\otimes i} \otimes \omega_{X}\right)
$$

We obtain the required vanishings by applying Proposition 4.4 with $\mathscr{E}_{1}=T_{X}$, $\mathscr{E}_{2}=\mathscr{F}^{*} \otimes \mathscr{A}^{-1}$, and $\mathscr{L}=\mathscr{A}^{\otimes i} \otimes \omega_{X}$.

In order to prove Theorem 4.3, we will compute several cohomology groups of special vector bundles on $\mathbb{P}^{n}$. We start by recalling Bott's formulae.

4.5 (Bott's formulae). Let $n$ be a positive integer, $k$ and $p$ non-negative integers, and $s$ an arbitrary integer. Then

$$
h^{p}\left(\mathbb{P}^{n}, \Omega_{\mathbb{P}^{n}}^{k}(s)\right)= \begin{cases}\left(\begin{array}{c}
s+n-k \\
s
\end{array}\right)\left(\begin{array}{c}
s-1 \\
k
\end{array}\right) & \text { for } p=0,0 \leq k \leq n \text { and } s>k, \\
1 & \text { for } s=0 \text { and } 0 \leq k=p \leq n, \\
\left(\begin{array}{c}
-s+k \\
-s
\end{array}\right)\left(\begin{array}{c}
-s-1 \\
n-k
\end{array}\right) & \text { for } p=n, 0 \leq k \leq n \text { and } s<k-n, \\
0 & \text { otherwise. }\end{cases}
$$

In addition to Proposition 4.4, we will need the following result.

Proposition 4.6 ([Man96, p.404]). Let $\mathscr{E}$ be a vector bundle on $\mathbb{P}^{n}$, and $p_{0}$ an integer. Suppose that

$$
H^{p}\left(\mathbb{P}^{n}, \omega_{\mathbb{P}^{n}} \otimes \mathscr{E}(s)\right)=0
$$

for every non-negative integer $s$ and every $p \geq p_{0}$. Then

$$
H^{p}\left(\mathbb{P}^{n}, \Omega_{\mathbb{P}^{n}}^{k} \otimes \mathscr{E}(s)\right)=0
$$

for any non-negative integers $s$ and $k$, and every $p \geq p_{0}$. 
We are now ready to establish the desired vanishing.

Proposition 4.7. Let $\mathscr{E}$ be a vector bundle of rank $k$ on $\mathbb{P}^{n}$, and suppose that one of the following conditions hold.

(1) $\mathscr{E}^{*}(k-n)$ is ample, or

(2) $\mathscr{E}$ decomposes as a direct sum of line bundles and $\mathscr{E}^{*}(k-n+1)$ is ample. Then

$$
H^{p}\left(\mathbb{P}^{n}, \bigwedge^{k} T_{\mathbb{P}^{n}} \otimes \Omega_{\mathbb{P}^{n}}^{i} \otimes S_{i-k}(\mathscr{E})\right)=0
$$

for any positive integers $i$ and $p$ satisfying $k+1 \leq i \leq n$ and $p<i-k+1$.

Proof. We may assume that $k \leq n$. By Serre duality,

$H^{p}\left(\mathbb{P}^{n}, \bigwedge^{k} T_{\mathbb{P}^{n}} \otimes \Omega_{\mathbb{P}^{n}}^{i} \otimes S_{i-k}(\mathscr{E})\right) \cong H^{n-p}\left(\mathbb{P}^{n}, \Omega_{\mathbb{P}^{n}}^{k} \otimes \bigwedge^{i} T_{\mathbb{P}^{n}} \otimes S_{i-k}\left(\mathscr{E}^{*}\right)(-n-1)\right)$.

Suppose first that $\mathscr{E}^{*}(k-n)$ is ample, and thus $\mathscr{E}^{*}(k-n-1)$ is nef. We write

$$
\bigwedge^{i} T_{\mathbb{P}^{n}} \otimes S_{i-k}\left(\mathscr{E}^{*}\right)(-n-1) \cong \bigwedge^{i}\left(T_{\mathbb{P}^{n}}(-1)\right) \otimes S_{i-k}\left(\mathscr{E}^{*}(k-n-1)\right) \otimes \mathcal{O}_{\mathbb{P}^{n}}(r),
$$

where $r=(n-k+1)(i-k)+i-n-1$. Since $i \geq k+1$, we get that $r \geq 1$, i.e., $\mathcal{O}_{\mathbb{P}^{n}}(r)$ is an ample line bundle. By applying Proposition 4.4 with $\mathscr{E}_{1}=T_{\mathbb{P}^{n}}(-1)$, $\mathscr{E}_{2}=\mathscr{E}^{*}(k-n-1)$ and $\mathscr{L}=\mathcal{O}_{\mathbb{P}^{n}}(r)$, we get:

$$
H^{n-p}\left(\mathbb{P}^{n}, \Omega_{\mathbb{P}^{n}}^{k} \otimes \bigwedge^{i} T_{\mathbb{P}^{n}} \otimes S_{i-k}\left(\mathscr{E}^{*}\right)(-n-1)\right)=0
$$

for any positive integers $i$ and $p$ satisfying $k+1 \leq i \leq n$ and $p<i-k+1$.

Suppose now that $\mathscr{E} \cong \bigoplus_{j=1}^{k} \mathcal{O}_{\mathbb{P}^{n}}\left(n-k+a_{j}\right)$, with $a_{j} \geq 0$. If $i>k+1$, then we write

$$
\bigwedge^{i} T_{\mathbb{P} n} \otimes S_{i-k}\left(\mathscr{E}^{*}\right)(-n-1) \cong \bigwedge^{i}\left(T_{\mathbb{P} n}(-1)\right) \otimes S_{i-k}\left(\mathscr{E}^{*}(k-n)\right) \otimes \mathcal{O}_{\mathbb{P} n}\left(r^{\prime}\right),
$$

with $r^{\prime}=(n-k)(i-k)+i-n-1 \geq 1$. The required vanishing then follows from Proposition 4.4 as above. From now on we assume that $i=k+1$ and $p=1$. Since cohomology commutes with direct sum, we must show that

$$
H^{n-1}\left(\mathbb{P}^{n}, \Omega_{\mathbb{P}^{n}}^{k} \otimes \bigwedge^{k+1} T_{\mathbb{P}^{n}}(a-k-1)\right)=0
$$

for every $a \geq 0$. By Bott's formulae we have

$$
\begin{gathered}
H^{n-1}\left(\mathbb{P}^{n}, \omega_{\mathbb{P}^{n}} \otimes \bigwedge^{k+1} T_{\mathbb{P}^{n}}(a-k-1+s)\right) \cong H^{n-1}\left(\mathbb{P}^{n}, \Omega_{\mathbb{P}^{n}}^{n-k-1}(a-k-1+s)\right)=0, \text { and } \\
H^{n}\left(\mathbb{P}^{n}, \omega_{\mathbb{P}^{n}} \otimes \bigwedge^{k+1} T_{\mathbb{P}^{n}}(a-k-1+s)\right) \cong H^{n}\left(\mathbb{P}^{n}, \Omega_{\mathbb{P}^{n}}^{n-k-1}(a-k-1+s)\right)=0
\end{gathered}
$$

for every non-negative integer $s$. Proposition 4.6 then implies (6). 
It is well known that the tangent bundle of $\mathbb{P}^{n}$ is simple, i.e., $H^{0}\left(\mathbb{P}^{n}, T_{\mathbb{P}^{n}} \otimes \Omega_{\mathbb{P}^{n}}^{1}\right) \cong$ $\mathbb{C}$ (see for instance OSS80, Lemma 4.1.2]). The next lemma shows that the same is true for exterior powers of $T_{\mathbb{P}^{n}}$, providing the last ingredient for the proof of Theorem 4.3

Lemma 4.8. Let $n$ be a positive integer, and $k \leq n$ a non-negative integer. Then $H^{0}\left(\mathbb{P}^{n}, \bigwedge^{k} T_{\mathbb{P}^{n}} \otimes \Omega_{\mathbb{P} n}^{k}\right) \cong \mathbb{C}$.

Proof. We start with the Euler sequence:

$$
0 \rightarrow \mathcal{O}_{\mathbb{P}^{n}}(-1) \rightarrow \mathcal{O}_{\mathbb{P}^{n}}^{\oplus n+1} \rightarrow T_{\mathbb{P}^{n}}(-1) \rightarrow 0
$$

By taking the $(k-i)$-th exterior power and twisting by $\Omega_{\mathbb{P}^{n}}^{k}(k-i), 0 \leq i \leq k-1$, we obtain the exact sequence

$$
0 \rightarrow \bigwedge^{k-i-1} T_{\mathbb{P}^{n}} \otimes \Omega_{\mathbb{P}^{n}}^{k} \rightarrow \Omega_{\mathbb{P}^{n}}^{k}(k-i)^{\oplus\left(\begin{array}{c}
n+1 \\
k-i
\end{array}\right)} \rightarrow \bigwedge^{k-i} T_{\mathbb{P}^{n}} \otimes \Omega_{\mathbb{P}^{n}}^{k} \rightarrow 0 .
$$

By Bott's formula, $H^{p}\left(\mathbb{P}^{n}, \Omega_{\mathbb{P}^{n}}^{k}(k-i)\right)=0$ for every $p \geq 0$, while $H^{k}\left(\mathbb{P}^{n}, \Omega_{\mathbb{P}^{n}}^{k}\right) \cong \mathbb{C}$. Hence, by taking cohomology of the above sequence we get

$$
\begin{aligned}
& H^{0}\left(\mathbb{P}^{n}, \bigwedge^{k} T_{\mathbb{P}^{n}} \otimes \Omega_{\mathbb{P}^{n}}^{k}\right) \simeq H^{1}\left(\mathbb{P}^{n}, \bigwedge^{k-1} T_{\mathbb{P}^{n}} \otimes \Omega_{\mathbb{P}^{n}}^{k}\right) \simeq \\
& H^{2}\left(\mathbb{P}^{n}, \bigwedge^{k-2} T_{\mathbb{P}^{n}} \otimes \Omega_{\mathbb{P}^{n}}^{k}\right) \simeq \cdots \simeq H^{k}\left(\mathbb{P}^{n}, \Omega_{\mathbb{P}^{n}}^{k}\right) \simeq \mathbb{C}
\end{aligned}
$$

Proof of Theorem 4.3. Let $\eta: \Omega_{\mathbb{P} n}^{k} \rightarrow\left(\bigwedge^{k} \mathscr{F}\right)^{*}$ be a Pfaff field of rank $k$ on $\mathbb{P}^{n}$, and suppose that $\operatorname{Sing}(\mathscr{F}) \subset \operatorname{Sing}(\eta)$. By Proposition 4.7, we have the required vanishing to apply Theorem 1.1 with $\mathscr{E}=\Omega_{\mathbb{P} n}^{1}$ and $\mathcal{G}=\mathscr{F}^{*}$. So we conclude that there is an endomorphism $\alpha$ of $\Omega_{\mathbb{P}^{n}}^{k}$ such that $\eta=\eta_{\mathscr{F}} \circ \alpha$. On the other hand, by Lemma 4.8. $\operatorname{End}\left(\Omega_{\mathbb{P}^{n}}^{k}\right) \cong H^{0}\left(\mathbb{P}^{n}, \bigwedge^{k} T_{\mathbb{P}^{n}} \otimes \Omega_{\mathbb{P}^{n}}^{k}\right) \cong \mathbb{C}$. Hence $\eta=\lambda \cdot \eta_{\mathscr{F}}$ for some $\lambda \in \mathbb{C}^{*}$.

Proof of Theorem 1.4. Let $r \in \mathbb{Z}$ be such that $\mathcal{O}_{\mathbb{P}^{n}}(r)=\operatorname{det}\left(\mathscr{F}^{*}\right) \otimes \omega_{\mathbb{P}^{n}}^{*}$. The con$\operatorname{dition} \operatorname{deg}\left(\mathscr{F}^{*}\right)>0$ implies that $r>n+1$. The distribution $\mathscr{F}$ induces a morphism $T_{\mathbb{P}^{n}} \rightarrow \mathcal{O}_{\mathbb{P}^{n}}(r)$, which corresponds to a nonzero section $\omega \mathscr{F} \in H^{0}\left(X, \Omega_{\mathbb{P} n}^{1}(r)\right)$.

By twisting the dual of the Euler sequence (7) by $\bigwedge^{i+1} T_{\mathbb{P}^{n}}(-i r+1), 1 \leq i \leq n-1$, we obtain the exact sequence

$$
0 \rightarrow \Omega_{\mathbb{P}^{n}}^{1} \otimes \bigwedge^{i+1} T_{\mathbb{P}^{n}}(-i r) \rightarrow \bigwedge^{i+1} T_{\mathbb{P}^{n}}(-i r-1)^{\oplus(n+1)} \rightarrow \bigwedge^{i+1} T_{\mathbb{P}^{n}}(-i r) \rightarrow 0
$$

By taking cohomology of the above sequence we get

$$
\begin{gathered}
\cdots \rightarrow H^{i-1}\left(\mathbb{P}^{n}, \bigwedge^{i+1} T_{\mathbb{P}^{n}}(-i r)\right) \rightarrow H^{i}\left(\mathbb{P}^{n}, \Omega_{\mathbb{P}^{n}}^{1} \otimes \bigwedge_{\mathbb{P}^{n}}(-i r)\right) \rightarrow \\
\rightarrow H^{i}\left(\mathbb{P}^{n}, \bigwedge^{i+1} T_{\mathbb{P}^{n}}(-i r-1)^{\oplus(n+1)}\right) \rightarrow \cdots
\end{gathered}
$$


By Bott's formulae, for $1 \leq i \leq n-1$,

$$
H^{i-1}\left(\mathbb{P}^{n}, \bigwedge^{i+1} T_{\mathbb{P}^{n}}(-i r)\right)=H^{i}\left(\mathbb{P}^{n}, \bigwedge^{i+1} T_{\mathbb{P}^{n}}(-i r-1)^{\oplus(n+1)}\right)=0
$$

Thus $H^{i}\left(\mathbb{P}^{n}, \Omega_{\mathbb{P}^{n}}^{1} \otimes \bigwedge^{i+1} T_{\mathbb{P}^{n}}(-i r)\right)=0$ for $1 \leq i \leq n-1$.

Let $\omega \in H^{0}\left(X, \Omega_{\mathbb{P} n}^{1}(r)\right)$ be such that $\left.\omega\right|_{\operatorname{Sing}(\mathscr{F})}=0$. It follows from Theorem 1.1 and Lemma 4.8 that there is $\alpha \in \mathbb{C}$ such that $\omega=\alpha \cdot \omega_{\mathscr{F}}$.

\section{REFERENCES}

[AD11] C. Araujo, S. Druel, On Fano foliations, Preprint arXiv:1112.4512 (2011).

[C-ACGLN04] O. Calvo-Andrade, D. Cerveau, L. Giraldo, A. Lins Neto, Irreducible components of the space of foliations associated to the affine Lie algebra, Ergodic Theory Dynam. Systems 24 (2004), no. 4, 987-1014.

[CO01] A. Campillo, J. Olivares, Polarity with respecto to a foliation and Cayley-Bacharach Theorems, J. Reine Angew. Math. 534 (2001), 95-118.

[CO03] A. Campillo, J. Olivares, On sections with isolated singularities of twisted bundles and applications to foliations by curves, Math. Res. Lett. 10 (2003), 651-658.

[CP08] F. Cukierman, J. V. Pereira, Stability of Foliations with Split Tangent Sheaf, Amer. J. of Math. 130 (2008), no. 2, 413-439.

[Cas91] G. Castelnuovo, Ricerche di geometria della retta nello spazio a quattro dimensioni, Atti R. Ist. Veneto Sc. 2 (1891), 855-901.

[CSV06] F. Cukierman, M. G. Soares, I. Vainsencher, Singularities of logarithmic foliations, Compositio Math. 142 (2006), 131-142.

[dMe00] A. S. de Medeiros, Singular foliations and differential p-forms, Ann. Fac. Sci. Toulouse Math. (6) 9 (2000), no. 3, 451-466.

[FF10] D. Faenzi, M.L. Fania, Skew-symmetric matrices and Palatini scrolls, Math. Ann. 347 (2010), 859-883.

[Fan30] G. Fano, Reti di complessi lineari dello spazio $S_{5}$ aventi una rigata assegnata di rettecentri, Rend. Reale Accad. Naz. Lincei, no. XI (1930), 227-232 .

[Eis95] D. Eisenbud, Commutative Algebra with a view toward Algebraic Geometry, Graduate Texts in Mathematics, vol. 150, Springer Verlag (1995).

[G-MK89] X. Gomez-Mont, G. Kempf, Stability of meromorphic vector fields in projective spaces. Comment. Math. Helv. 64 (1989), 462-473.

[GP-C10] L. Giraldo, A. J. Pan-Collantes, On the singular scheme of codimension one holomorphic foliations in $\mathbb{P}^{3}$, Internat. J. Math, 7 (2010), 843-858.

[Har78] R. Hartshorne, Stable vector bundles of rank 2 on $\mathbb{P}^{3}$, Math. Ann. 238, no. 3 (1978), 229-280.

[Jou79] J. P. Jouanolou, Equations de Pfaff algï $\ddot{2} \frac{1}{2}$ briques, Lecture Notes in Mathematics 708, Springer (1979).

[Man96] L. Manivel, Théorèmes d'annulation sur certaines variétés projectives, Comment. Math. Helv. 71, no. 3 (1996), 402-425.

[OSS80] C. Okonek, M. Schneider, H. Spindler, Vector bundles on complex projective spaces, Progress in Mathematics 3, Birkhaüser (1980).

[Pal01] F. Palatini, Sui sistemi lineari di complessi lineari di rette nello spazio a cinque dimensioni, Atti del R. Ist. Veneto di Scienze, Lettere e Arti, no. 60 (1901), 371-383.

[Pal03] F. Palatini Sui complessi lineari di rette negli iperspazi, Giorn. di Mat., no. 41 (1903), $85-96$. 
Carolina Araujo: impa, Estrada Dona Castorina 110, Rio de Janeiro, 22460-320, BRAZIL

E-mail address: caraujo@impa.br

Maurício Corrêa Jr: Universidade Federal de Viçosa, Departamento de Matemática, Avenida P.H. Rolfs, s/n, 36570-000, Viçosa-MG, Brazil

E-mail address: mauricio.correa@ufv.br 